\title{
Sistema de Raciocínio Baseado em Casos para Recomendação de Programa Alimentar
}

\author{
Dagoberto Telles Junior ${ }^{1}$, Viviane Carra Telles ${ }^{1}$, Fabiana Lorenzi ${ }^{1}$, Stanley Loh $^{1,2}$, Analucia \\ Schiaffino Morales De Franceschi ${ }^{1}$ \\ ${ }^{1}$ Universidade Luterana do Brasil (ULBRA) - UGC de Computação \\ Av. Farroupilha, 8.001 - Canoas, RS - CEP 92420-280 \\ ${ }^{2}$ Universidade Católica de Pelotas (UCPEL) - Escola de Informática \\ R. Félix da Cunha, 412 - Pelotas, RS, CEP 96010-000
}

dtjrf311 AT uol.com.br, vivianecarra AT uol.com.br, fabilorenzi AT gmail.com, sloh AT terra.com.br, analuciadefranceschi AT gmail.com

\begin{abstract}
Resumo
Este artigo apresenta um sistema para recomendação de dietas alimentares que utiliza a técnica de Raciocínio Baseado em Casos (RBC). O sistema é aplicado ao domínio da especialidade médica de nutrição, sendo que os casos são representados por pacientes. Ao longo do trabalho, são apresentadas as estratégias para a definição dos casos e do método de recuperação usando avaliações de similaridade. $O$ benefício principal do sistema é apoiar a tomada de decisão do profissional de nutrição, fornecendo informações precisas sobre casos semelhantes registrados no histórico. O sistema foi validado com casos reais. $\mathrm{O}$ artigo ainda discute questões técnicas relativas à aplicação de $\mathrm{RBC}$, suas dificuldades, vantagens e cuidados, embasados num caso prático.
\end{abstract}

Palavras-chaves: Raciocínio Baseado em Casos; Sistemas de Recomendação, Dieta Alimentar.

\begin{abstract}
This paper presents a nutritional recommendation system based on a Case-Based Reasoning (CBR) technique. The cases have been represented by patients and then applied to support nutritional professionals in decision-making processes for determining diet programs. The system is based on historical cases, registered by the professional in his office. The evaluation of the system usage in actual cases is presented. The paper also discusses technical issues related to CBR application, such as the difficulties, the advantages, the mistakes and the attentions which have been observed in an actual application.
\end{abstract}

Keywords: Case-Based Reasoning; Recommender Systems; Nutritional Diet.

\section{INTRODUÇÃo}

Os avanços da medicina no conhecimento do corpo humano e a preocupação crescente com a aparência e bem-estar têm feito com que se tenha mais cuidado com a saúde e em especial com a alimentação.

Sabe-se da influência de maus hábitos alimentares no desenvolvimento de doenças. Neste sentido, a nutrição exerce um papel muito importante para melhorar a qualidade de vida das pessoas.

A nutrição surgiu a partir da necessidade de conhecer os nutrientes fundamentais para o bom funcionamento do corpo humano, uma vez que durante seu funcionamento, elementos são destruídos (catabolismo) e construídos (anabolismo). Em outras palavras, é importante preocupar-se com a qualidade e a quantidade dos alimentos que são ingeridos, pois desta forma dá-se subsídio para que o organismo realize suas funções de forma adequada [19].

Para determinar a quantidade diária necessária de alimentos que um indivíduo necessita, consideramse fatores elementares como sexo, peso, idade e atividade física que esta pessoa realiza. Por outro lado, observou-se a existência e influência de outros fatores menos elementares, no sucesso de programas alimentares. Por vezes, pacientes com a mesma biometria não conseguem atingir o resultado esperado, acabando com as formulações prontas e dando espaço a 
técnicas heurísticas. A identificação destes critérios exige do especialista, ferramentas dinâmicas que suportem a busca e descoberta de conhecimento.

Este artigo apresenta o sistema DietSysNet, concebido para apoiar profissionais de nutrição (chamados "nutricionistas") na tarefa de prescrever dietas alimentares, através do emprego das técnicas de Raciocínio Baseado em Casos (RBC, ou Case-Based Reasoning - CBR). RBC é uma técnica de Inteligência Artificial que permite modelar o raciocínio e o pensamento humano e construir sistemas computacionais inteligentes que utilizam o aprendizado de experiências do passado para a solução de problemas atuais [10].

Basicamente, o sistema registra dados clínicos e pessoais de pacientes para os quais já foram feitas prescrições corretamente validadas e utiliza esta base de casos anteriores para reaproveitar prescrições alimentares para pacientes novos com características similares. A cada caso novo, o sistema procura na base por casos semelhantes e recomenda para o nutricionista a mesma prescrição. $\mathrm{O}$ sistema também permite que o nutricionista adapte as prescrições de dietas, gerando assim novos casos para serem incluídos na base de dados que servirá para comparação.

O sistema também dispõe de um mecanismo de acompanhamento do tratamento, onde o paciente fornece pela Internet informações a respeito do cumprimento de seu programa alimentar, tais como: peso, ocorrência de desvio alimentar, efeito colateral percebido, alergia, tontura, última medição de pressão arterial ou de glicose, colesterol, triglicerídeos, ácido úrico, exames de sangue, de urina, etc. Tais informações possibilitam ao especialista reavaliar os valores nutricionais da dieta e manter-se informado sobre o andamento do tratamento. Trata-se de uma alternativa ágil de proporcionar ao especialista "feedback" sobre o tratamento recomendado.

Este artigo está organizado da seguinte forma: o capítulo 2 apresenta o domínio do sistema. O capítulo 3 mostra as etapas de desenvolvimento do sistema RBC. No capítulo 4 é apresentado o DietSysNet. O capítulo 5 explica como foi efetivada a validação do sistema e o capítulo 6 apresenta os trabalhos semelhantes. Finalmente, são apresentadas as conclusões obtidas e as devidas referências.

\section{O DOMÍNIO Do SISTEMA}

O domínio do sistema está baseado no cotidiano do profissional de nutrição. O nutricionista coleta através de entrevistas informações sobre o paciente tais como: dados de identificação, peso, altura, peso pretendido, índice de massa corporal (IMC); histórico clínico e familiar; hábitos alimentares; doenças que está tratando ou que já enfrentou; alimentos que consome: líquidos, frituras, sal, gorduras, guloseimas, alimentos industrializados; utilização de medicamentos; consumo de álcool ou cigarro.

As principais patologias relacionadas à alimentação, tais como a obesidade, colesterol alto, diabete e hipertensão, também devem ser relacionadas a cada paciente para o entendimento das restrições alimentares que as patologias impõem.

Em seguida, inicia-se a avaliação do perfil do paciente e são executados os chamados cálculos de dietoterapia. Também são anotados possíveis efeitos colaterais percebidos como tonturas, dores de cabeça, irritabilidade, alergias alimentares, dentre outros. Caso o paciente tenha realizado exames relevantes (sangue, glicose ou pressão arterial), os resultados também ficam registrados para um acompanhamento da evolução do quadro do paciente.

De posse destas informações, o nutricionista elabora a prescrição, interpelando o paciente sobre preferências alimentares e condições disponíveis para cada refeição. Nela estão os alimentos, quantidades e horários que devem ser consumidos. A verificação quanto ao valor calórico da prescrição é feita através do somatório de valores individuais de cada alimento, multiplicado pela sua quantidade.

A prescrição divide o dia do paciente em seis refeições: desjejum, colação, almoço, lanche, janta e ceia. A lista das refeições e dos alimentos é mostrada ao paciente, bem como as substituições possíveis. Substituições são alimentos alternativos aos prescritos, que por possuírem o mesmo valor nutricional, permitem ao paciente adaptar a dieta às suas preferências ou condições econômicas.

A cada nova consulta, o nutricionista tem que refazer diversos cálculos encadeados e consultar tabelas. Por ser manual, a chance de ocorrerem erros neste processo é grande. Além disto, a consulta dos parâmetros possui muitas ponderações não formais. $\mathrm{O}$ conhecimento adquirido e acumulado através deste processo é confiado à memória do profissional.

O nutricionista já reutiliza soluções baseadas no aprendizado obtido com experiências anteriores para determinar a dieta do paciente que está atendendo, mas sempre informalmente e com pouca sistemática. Este é claramente um domínio candidato a uma solução baseada em RBC.

\section{A TÉCNICA DE RBC}

O ser humano naturalmente utiliza-se da experiência de uma situação já conhecida para a resolução de um problema. Aproveitar conhecimentos adquiridos em situações passadas é algo feito constantemente, muitas vezes de forma inconsciente.

Embasado neste modelo de aprendizado humano surgiu o RBC, que é uma técnica de inteligência artificial que pode ser vista de duas maneiras: uma metodologia para modelar o raciocínio e o pensamento humano ou uma metodologia para construir sistemas computacionais inteligentes [20]. O paradigma do RBC busca sistematizar o emprego do aprendizado de experiências do passado para a solução de problemas atuais. O ciclo de funcionamento desta técnica compreende as seguintes etapas:

1. Armazenamento de casos: nesta etapa, a estrutura 
dos casos é modelada e os casos a serem utilizados no sistema são armazenados conforme o modelo, formando a Base de Casos (BC); para esta etapa, é necessário determinar os atributos para a caracterização dos problemas e a forma como a solução será representada;

2. Recuperação: nesta fase procura-se na BC aqueles casos que mais se assemelham com o problema proposto. Para esta pesquisa, é necessário um método de avaliação de similaridade entre os casos.

3. Reutilização: uma vez identificado o caso semelhante supõe-se que a solução adotada também servirá para a situação atual.

4. Revisão: nesta fase, as eventuais diferenças que podem existir entre os casos são ajustadas através da adaptação da solução recuperada. Esta fase da revisão realiza-se através de simulações ou até mesmo na aplicação prática e assemelha-se com o processo cognitivo humano, onde se busca confirmar a solução do problema [20].

5. Retenção: nesta fase, armazena-se a experiência ou partes interessantes do novo problema para reutilização. Através da retenção estende-se a base de conhecimento, uma vez que contempla as adaptações realizadas, aprimorando assim o conhecimento.

\subsection{A Representação de Casos em RBC}

Os sistemas de RBC utilizam casos para representar o conhecimento. Um caso representa o conhecimento do especialista na solução de determinados problemas [12][13][14]. Este tipo de representação é considerado um caso particular do raciocínio por analogia, que busca uma solução semelhante ao problema caso ele já tenha sido resolvido [2].

Um caso consiste então em uma experiência real onde o problema proposto já foi resolvido, procurando-se representar o problema através de atributos. Estes atributos, juntamente com seus valores devem identificar da forma mais distinta possível o contexto e o conteúdo do problema [10]. A definição de caso é parte importante de um sistema de RBC, pois um problema mal definido ou identificado poderá gerar uma recomendação não adequada.

Alguns exemplos de utilização de casos são: o médico ao atender um paciente analisa os sintomas apresentados para formular um diagnóstico da doença e apresenta a medicação indicada para a cura; um advogado consulta bancos de processos encerrados, procurando por processos semelhantes ao que ele está defendendo para poder munir-se de argumentação.

Segundo Kolodner [10], a representação de um caso compreende três componentes básicos:

1. Descrição do problema: a definição das características que contribuem para a identificação do problema. Estas podem ser nomes, números, textos, funções representativas para a correta descrição e contextualização do problema.

2. Descrição da solução: corresponde à solução recuperada pelo sistema para o problema proposto, devidamente validada e descrita.

3. Conclusão: também chamada de resultado, consiste em uma avaliação da solução recomendada durante a sua aplicação, demonstrando os resultados obtidos. Recomendações quanto a futuras utilizações desta solução podem fazer parte da conclusão e conferem maior qualidade e confiabilidade à $\mathrm{BC}$.

\subsection{A Recuperação de Casos em RBC}

A etapa de recuperação de casos inicia com uma descrição de problema e finaliza quando um melhor caso for encontrado. O sistema procura na base de casos, o caso mais parecido com o novo problema. Para julgar qual o caso armazenado na base é parecido ou igual ao novo problema, é preciso medir a similaridade (semelhança) entre eles.

Para calcular esta similaridade, normalmente os sistemas de RBC utilizam o Algoritmo do vizinho mais próximo (nearest neighbor), representado na fórmula abaixo:

$$
d_{(q, c)}=\sqrt{\sum_{f=1}^{n} w_{f} * \operatorname{sim}\left(q_{f}, c_{f}\right) 2}
$$

onde $q$ é o novo problema, $c$ é o caso da base, $f$ é o atributo, $w$ o peso do atributo e sim é a função de similaridade entre o novo problema e o caso. $\mathrm{Na}$ função de similaridade, podem ser utilizadas funções como a Distância Euclidiana, Distância de Hamming Coeficiente do Co-seno ou Coeficiente de Jaccard [2].

A função de similaridade de atributos numéricos é calculada através da distância entre os valores. Em atributos simbólicos (que admitem somente valores "sim" ou "não", por exemplo), utilizase a similaridade local, onde a distância é igual a 1 se os valores forem iguais e 0 se forem diferentes.

Já no caso de atributos com escala (faixa etária, por exemplo), cada valor possível deve ter sua distância calculada em relação aos demais valores, resultando numa matriz de similaridade entre os valores possíveis.

Entretanto, há casos onde esta determinação de distância pode não ser tão trivial. É o caso de atributos com valores nominais (bairro e cidade, por exemplo). Este é o tipo de atributo mais difícil de ser usado numa função de similaridade. As opções são definir uma escala de forma arbitrária ou então considerar como uma escala binária onde valores iguais resultam em $1 \mathrm{e}$ valores diferentes resultam em 0 .

O resultado da recuperação de casos pode ser um ranking de casos, ordenados por similaridade (os mais similares primeiro ou no topo). Pode-se utilizar um limiar para filtrar somente casos com similaridade acima deste limiar. Isto diminuirá o volume de resultados e não gera sobrecarga de informações para quem está tomando a decisão.

\subsection{Reutilização, Revisão e Retenção de}




\section{Casos}

O reuso dos casos pode ser realizado após a recuperação. $\mathrm{O}$ usuário que toma a decisão opta por aceitar ou não a recomendação do sistema. Este usuário pode avaliar os casos mais similares, apresentados pelo ranking, e decidir reutilizar algum outro no topo do ranking, ao invés do caso mais similar.

Além disto, o usuário pode decidir por ajustar a solução recomendada pelo sistema. Isto é comum quando não há um caso $100 \%$ similar.

O processo de revisão dos casos recomendados, também conhecido como adaptação de casos, permite incorporar conhecimento especialista humano ao processo, principalmente quando o domínio é muito complexo e o sistema de RBC não consegue capturar todo o conhecimento tácito.

O usuário de um sistema de RBC pode então decidir incorporar à $\mathrm{BC}$ o novo caso com a solução adaptada. Isto faz com que a $\mathrm{BC}$ cresça com qualidade e gere casos diversos. A conseqüência é que na recuperação de casos, há maiores chances de se encontrar um caso similar ao que está sendo testado (aumenta a probabilidade de casos similares). Este processo, chamado de retenção, é importante para gerar casos semelhantes, mas com pequenas diferenças e é uma das vantagens da abordagem de RBC, pois representa o aprendizado do sistema. Assim, fica mais fácil encontrar casos com maior semelhança para um caso que precise de uma solução específica.

É também possível incorporar casos fictícios à $\mathrm{BC}$. Estes casos não representam um indivíduo real, mas representam um grupo de indivíduos. Isto aumenta a diversidade de casos e melhora as chances na hora da recuperação.

\section{O Sistema DietSysNet}

Foi desenvolvido um sistema, chamado de DietSysNet, para auxiliar os profissionais da área de nutrição (chamados de nutricionistas) no momento de decidir por um programa alimentar para os pacientes.

O objetivo não é substituir o especialista humano, no entanto, pretende-se dar subsídios para uma melhor decisão através do fornecimento de informações precisas. Desta forma, o resultado do sistema é uma recomendação de prescrições possíveis, conforme o histórico de pacientes registrados no sistema. Estas prescrições recomendadas são as que foram utilizadas com sucesso em casos semelhantes no passado, ou seja, prescrições que deram resultados positivos para pacientes com características semelhantes ao paciente que está sendo analisado.

No sistema desenvolvido, os casos são representados por pacientes que receberam prescrições. Cada caso é representado por um problema (o perfil do paciente) e uma solução (a prescrição alimentar dada pelo especialista para cada paciente).

Este sistema adota as cinco etapas previstas para o funcionamento de um sistema de RBC mencionadas anteriormente (armazenamento dos casos, recuperação, reutilização, revisão e retenção).

A seguir, são apresentados os conceitos tecnológicos empregados para a construção do sistema, além da descrição do projeto e a devida implementação do sistema.

\subsubsection{Definição dos Casos no DietSysNet}

No sistema DietSysNet, um caso é representado por um paciente específico. Cada caso é dividido em um problema e uma solução.

O problema foi representado pelo cadastro de do paciente, composto por seus dados de identificação e um perfil que responde a uma seqüência de atributos definidos. Já a solução é representada pela prescrição alimentar e as recomendações dadas em seu tratamento.

Os quadros 1a e $1 \mathrm{~b}$ apresentam os atributos utilizados para caracterizar o perfil do paciente. Além disto, os mesmos quadros apresentam os pesos dos atributos e os tipos de respostas possíveis (valores possíveis ou faixas de valores para os atributos). A adoção de grupos de valores para os atributos simplificou a recuperação dos casos e facilitou o cálculo de similaridade local. Os pesos foram definidos por um nutricionista e serão detalhados de forma mais adequada na seção 4.3.1.

O sistema é genérico o suficiente para permitir expandir o conhecimento através da inclusão de novos atributos. Entretanto, se isto for realizado, os casos já armazenados também deverão ter valores registrados para estes novos atributos. Cada novo atributo deverá ter associado um grupo de valores possíveis e um peso. 
Quadro 1a - Atributos dos casos

\begin{tabular}{|c|c|c|}
\hline $\begin{array}{l}\text { Descrição da } \\
\text { Característica }\end{array}$ & Peso & Respostas \\
\hline $\begin{array}{l}\text { Freqüência do } \\
\text { Consumo } \\
\text { de Álcool }\end{array}$ & 3 & $\begin{array}{c}\text { Nunca } \\
\text { Raro } \\
\text { Periódico } \\
\text { Freqüente }\end{array}$ \\
\hline Anemia & 1 & Sim/Não \\
\hline $\begin{array}{c}\text { Problemas Digestivos } \\
\text { ou Perda de Apetite }\end{array}$ & 1 & Sim/Não \\
\hline $\begin{array}{c}\text { Irritabilidade, Insônia e } \\
\text { Lapsos de Memória } \\
\end{array}$ & 1 & Sim/Não \\
\hline Convalescença & 1 & Sim/Não \\
\hline Vegetariano & 4 & Sim/Não \\
\hline Cãibras Musculares & 1 & Sim/Não \\
\hline $\begin{array}{l}\text { Problemas Renais } \\
\text { Dificuldades na } \\
\text { Cicatrização }\end{array}$ & $\begin{array}{l}4 \\
1\end{array}$ & $\begin{array}{l}\text { Sim/Não } \\
\text { Sim/Não }\end{array}$ \\
\hline Hipoglicemia & 1 & Sim/Não \\
\hline $\begin{array}{l}\text { Freqüência do } \\
\text { Consumo de Água }\end{array}$ & 5 & $\begin{array}{c}\text { Nunca } \\
\text { Raro } \\
\text { Periódico } \\
\text { Freqüente } \\
\end{array}$ \\
\hline $\begin{array}{c}\text { Utiliza Medicamentos } \\
\text { que influem nos } \\
\text { Nutrientes }\end{array}$ & 1 & Sim/Não \\
\hline $\begin{array}{c}\text { Usuário } \\
\text { de Drogas } \\
\end{array}$ & 1 & Sim/Não \\
\hline Hipertensão & 10 & Sim/Não \\
\hline Colesterol & 10 & Sim/Não \\
\hline Diabetes & 10 & Sim/Não \\
\hline Sexo & 10 & $\begin{array}{r}\text { Feminino } \\
\text { Masculino }\end{array}$ \\
\hline Faixa Etária & 6 & $\begin{array}{c}10-18 \\
18-30 \\
30-60 \\
>\text { de } 60 \\
\end{array}$ \\
\hline $\begin{array}{l}\text { Tempo Dedicado } \\
\text { às Refeições }\end{array}$ & 3 & $\begin{array}{l}\text { Nenhum } \\
\text { Pouco } \\
\text { Normal } \\
\text { Bastante }\end{array}$ \\
\hline $\begin{array}{c}\text { Histórico de } \\
\text { Hipertensão, } \\
\text { Colesterol, Diabetes, } \\
\text { Obesidade }\end{array}$ & 2 & Sim/Não \\
\hline
\end{tabular}

Quadro 1b - Atributos dos casos

\begin{tabular}{|c|c|c|}
\hline $\begin{array}{l}\text { Descrição da } \\
\text { Característica }\end{array}$ & Peso & Respostas \\
\hline Nível de Atividade & 5 & $\begin{array}{l}\text { Repouso } \\
\text { Leve } \\
\text { Moderado } \\
\text { Ativo }\end{array}$ \\
\hline $\begin{array}{l}\text { Faixa de VET } \\
\text { (valor energético } \\
\text { teórico) }\end{array}$ & 10 & $\begin{array}{c}\text { Valores de uma } \\
\text { tabela auxiliar }\end{array}$ \\
\hline Fumante & 2 & Sim/Não \\
\hline Alergia alimentar & 2 & Sim/Não \\
\hline Utilizou anorexígenos & 2 & Sim/Não \\
\hline $\begin{array}{c}\text { Consumo chá, café ou } \\
\text { chimarrão }\end{array}$ & 2 & Sim/Não \\
\hline Dieta anterior & 2 & Sim/Não \\
\hline Dentes em bom estado & 2 & Sim/Não \\
\hline $\begin{array}{c}\text { IMC } \\
\text { (índice de massa } \\
\text { corporal) }\end{array}$ & 10 & $\begin{array}{c}\text { Valores de uma } \\
\text { tabela auxiliar }\end{array}$ \\
\hline $\begin{array}{l}\text { Consumo de } \\
\text { Refrigerantes }\end{array}$ & 4 & $\begin{array}{l}\text { Nenhum } \\
\text { Pouco } \\
\text { Normal } \\
\text { Bastante }\end{array}$ \\
\hline Nível de Ansiedade & 2 & $\begin{array}{l}\text { Nenhum } \\
\text { Pouco } \\
\text { Normal } \\
\text { Bastante } \\
\end{array}$ \\
\hline $\begin{array}{l}\text { Hábito intestinal, } \\
\text { hábito urinário, }\end{array}$ & 2 & $\begin{array}{l}\text { Nenhum } \\
\text { Pouco } \\
\text { Normal } \\
\text { Bastante }\end{array}$ \\
\hline Nível de Ansiedade & 2 & $\begin{array}{l}\text { Nenhum } \\
\text { Pouco } \\
\text { Normal } \\
\text { Bastante } \\
\end{array}$ \\
\hline Utiliza adoçante & 2 & $\begin{array}{l}\text { Nenhum } \\
\text { Pouco } \\
\text { Normal } \\
\text { Bastante } \\
\end{array}$ \\
\hline $\begin{array}{c}\text { consumo de Frituras, } \\
\text { Guloseimas, Sal, } \\
\text { Salada, Frutas, } \\
\text { Embutidos }\end{array}$ & 2 & $\begin{array}{l}\text { Nenhum } \\
\text { Pouco } \\
\text { Normal } \\
\text { Bastante }\end{array}$ \\
\hline
\end{tabular}




\subsection{Geração da Base de Casos}

A Base de Casos (BC) é formada pelos perfis dos pacientes já atendidos como também pelas respectivas prescrições aplicadas pelo nutricionista a cada caso. A $\mathrm{BC}$ é o elemento fundamental do sistema, ou seja, de onde serão extraídos os casos similares. Assim, uma $\mathrm{BC}$ com pequena quantidade de casos tende a indicar um número menor de semelhanças e provavelmente terá um índice médio baixo de assertividade.

No DietSysNet, a BC iniciou com o elenco de pacientes de um consultório, em número de 40 casos, que foram registrados no sistema com dados de perfis e prescrição.

Quando há casos novos cuja similaridade com casos da BC não seja adequada ou suficiente, podem ser criados perfis mestres, ou seja, modelos básicos que contemplarão as situações ainda não atendidas. Os perfis mestres baseiam-se no conhecimento do especialista do domínio sobre os tipos de paciente que são esperados. Este processo de formação de uma base contendo os casos mais corriqueiros proporciona ao sistema operar um maior número de tipos de problemas (diversidade) e conseqüentemente obter melhor desempenho no momento da recuperação de casos, já que aumenta a probabilidade de encontrar casos mais semelhantes.

\subsection{Recuperação dos casos}

A recuperação consiste na busca pelo caso mais similar ao apresentado dentre aqueles armazenados na BC. Segundo Wangenhein [20], para realizar esta recuperação, é necessário casar a descrição do problema atual com os problemas armazenados na BC, aplicando uma medida de similaridade. Para aplicar esta medida, é calculado um valor de similaridade (VS) para cada caso da base. Apresenta-se melhor a avaliação de similaridade na seção 4.3.2. De um modo geral a recuperação contempla três etapas:

1. Qualificação do caso - onde o caso atual é contextualizado na forma definida para a $\mathrm{BC}$;

2. Comparação entre casos - onde ocorre o casamento do novo caso aos existentes obtendo os casos suficientemente similares;

3. Seleção de caso - onde o melhor caso é escolhido dentre os similares.
Os sistemas RBC devem demonstrar sua praticidade oferecendo rapidez na tarefa de recuperação de casos. Para isso é muito importante a definição de um método eficiente que cumpra devidamente sua função de organizadores da BC. Nota-se que, desde a inclusão, é necessário preocupar-se com a melhor maneira de preparar a $\mathrm{BC}$ para $\mathrm{o}$ processo de recuperação.

Dentre os diversos métodos existentes para recuperação, optou-se pela Recuperação em Dois Níveis como a mais adequada para o projeto. O princípio básico do método consiste em aperfeiçoar a etapa de comparação, reduzindo o número de casos avaliados.

Este processo é inspirado originalmente no modelo de aprendizado cognitivo MAC/FAC. Tal modelo tenta tornar a recuperação mais ágil aplicando dois níveis de recuperação: (a) a pré-seleção de candidatos, onde uma lista de casos candidatos é obtida por intermédio de uma comparação simples, mas com mínimas chances de erro; e (b) a ordenação dos candidatos, onde os casos pré-selecionados são submetidos a uma comparação mais complexa e detalhada, usando similaridade, e gerando um ranking de similaridade.

No DietSysNet, para a pré-seleção, foram identificados atributos chamados de primeiro nível. Estes atributos são os mais significativos e deverão ter valores iguais para um caso poder ser recuperado. Isto evita comparações desnecessárias. $\mathrm{O}$ atributo sexo foi definido juntamente com o especialista como sendo a única característica que devia ser usada na pré-seleção.

A figura 1 mostra a tela do sistema com o resultado da recuperação de casos (após a avaliação de similaridades). Vê-se o ranking de casos resultantes, sendo que cada paciente (caso) indicado pelo sistema como similar ao sendo avaliado possui um percentual de similaridade.

Na mesma tela, o sistema apresenta os valores dos atributos para o caso sendo testado e para o caso mais similar. Isto permite explicar ao usuário do sistema a similaridade entre os casos e permite a comparação dos perfis.

A interpretação destes resultados indica a existência de um paciente (caso) na $\mathrm{BC}$ com índice de $92,67 \%$ de similaridade. Nota-se que, em apenas 4 atributos, não há similaridade local igual a 1 (consumo de guloseimas, consumo de frutas, consumo de refrigerantes e se é fumante ou não).

Nesta mesma tela, pode-se ver no alto à direita o limiar mínimo para apresentação de casos (configurado em 85\%). 


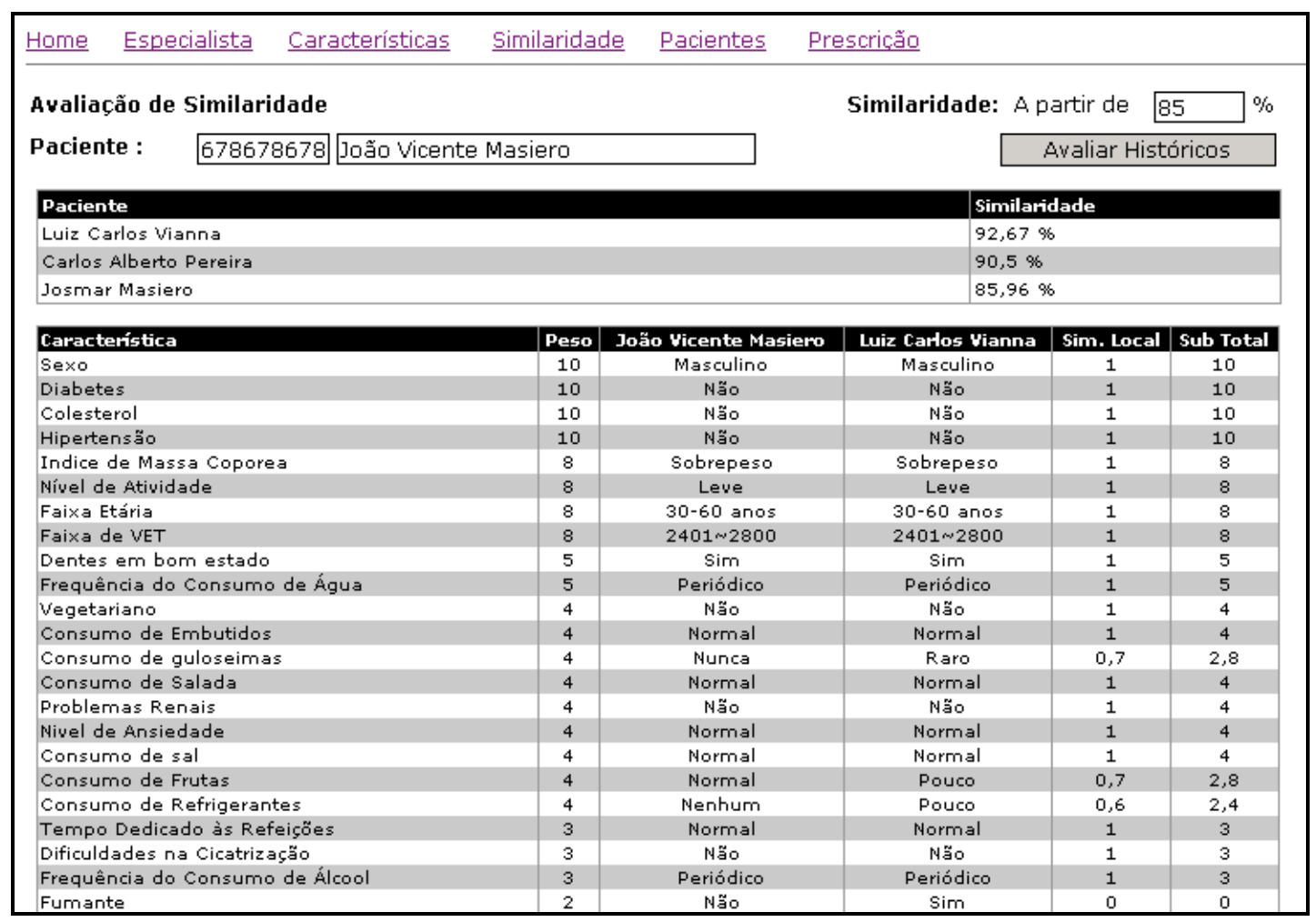

Figura 1 - Tela com Resultado da Recuperação de Casos

\subsubsection{Atribuição de pesos aos atributos}

Utilizam-se pesos nos atributos para o cálculo da medida de similaridade entre os casos. É necessário associar um peso para cada atributo da base, o qual significa o grau de importância daquela característica dentre os outros atributos que contextualizam o caso.

Neste sistema, os pesos foram definidos por um especialista em nutrição, após algumas simulações no sistema com dados fictícios e reais.

Os valores de pesos variam de 1 (ou menos importante) a 10 (ou mais importante).

\subsubsection{Avaliação de Similaridade}

A medida de similaridade é uma avaliação numérica que indica o quanto um caso é parecido com o outro. $\mathrm{O}$ resultado é dado em percentual. Existem 2 tipos de similaridade: a global e a local.

Para avaliar a similaridade local entre os casos (problemas), é necessário avaliar a similaridade entre os atributos de cada caso. Para tanto, toma-se o valor do atributo $\mathrm{X}$ no caso 1 e compara-se com o valor do mesmo atributo $\mathrm{X}$ no caso 2 .

Foram definidos valores de similaridade local entre os possíveis valores de resposta para cada atributo. Estes valores de similaridade (VS) foram estudados e definidos pelo especialista no domínio, após algumas simulações no sistema com dados fictícios e reais.. O resultado do VS é dado por um número real que varia entre 0 para respostas diferentes e 1 para respostas idênticas.

Para os atributos que possuem no máximo duas respostas (por exemplo, "sim" e "não"), o VS é igual a 1 se forem iguais e 0 caso contrário.

Para os atributos com mais de 2 respostas possíveis, é necessário um outro tipo de avaliação, onde se compara a resposta do atributo do problema com a resposta deste mesmo atributo no caso préselecionado recuperando-se o valor de similaridade (VS) correspondente.

A tabela 1 apresenta os valores de similaridade locais de atributos que possuem valores relativos à freqüência (nunca, raro, periódico, freqüente). Nota-se que a similaridade local entre o valor "nunca" e o valor "periódico" é 0.2 e vice-versa. Normalmente, as matrizes com os VS locais são simétricas. Novamente o especialista foi responsável pela definição das similaridades locais para cada tipo de atributo.

Posteriormente, é efetuado o cálculo do valor de similaridade global que utiliza a média ponderada. $\mathrm{O}$ cálculo é feito da seguinte maneira: soma-se o VS local de cada atributo multiplicado pelo peso do atributo e divide-se este resultado pelo somatório dos pesos. O sistema ordena os casos conforme o VS global apresentando-os na tela como solução para o problema. As tabelas 2 e 3 apresentam exemplos de cálculo de similaridade global.

A figura 2 apresenta a tela onde os VS locais são definidos no sistema. 
Tabela 1 - VS local para respostas com Freqüência

\begin{tabular}{c|c|c|c|c}
\hline Freqüência & Nunca & Raro & Periódico & Freqüente \\
\hline Nunca & 1.0 & 0.8 & 0.2 & 0.2 \\
\hline Raro & 0.8 & 1.0 & 0.2 & 0.2 \\
\hline Periódico & 0.2 & 0.2 & 1.0 & 0.8 \\
\hline Freqüente & 0.2 & 0.2 & 0.8 & 1.0 \\
\hline
\end{tabular}

Tabela 2 - Exemplo 1 cálculo de similaridade

\begin{tabular}{|l|c|c|c|c|}
\hline \multicolumn{1}{|c|}{ Atributos } & Peso & $\begin{array}{c}\text { Caso } \\
\text { atual }\end{array}$ & $\begin{array}{c}\text { Caso 1 da } \\
\text { BC }\end{array}$ & $\begin{array}{c}\text { VS } \\
\text { local }\end{array}$ \\
\hline Consumo água & 3 & Periódico & Nunca & 0,2 \\
\hline Consumo álcool & 3 & Nunca & Periódico & 0,2 \\
\hline Faixa etária & 8 & $30-60$ & $30-60$ & 1,0 \\
\hline Nível atividade & 10 & Leve & Moderado & 0,8 \\
\hline Valor do VET & 10 & $2001-2400$ & $2001-2400$ & 1,0 \\
\hline \multicolumn{6}{|c|}{ VS global: $\mathbf{( 3 * 0 , 2}+\mathbf{3} * \mathbf{0 , 2}+\mathbf{8 * 1 , 0 + 1 0 * 0 , 8}+$} \\
$\mathbf{1 0 * 1 , 0 )} / \mathbf{3}+\mathbf{3}+\mathbf{8}+\mathbf{1 0}+\mathbf{1 0}=\mathbf{0 , 8}$ \\
\hline
\end{tabular}

Tabela 3 - Exemplo 2 cálculo de similaridade

\begin{tabular}{|c|c|c|c|c|}
\hline Atributos & Peso & $\begin{array}{l}\text { Caso } \\
\text { atual }\end{array}$ & $\begin{array}{c}\text { Caso } 2 \text { da } \\
\text { BC }\end{array}$ & $\begin{array}{c}\mathrm{VS} \\
\text { local }\end{array}$ \\
\hline Consumo água & 3 & Periódico & Raro & 0,2 \\
\hline Consumo álcool & 3 & Nunca & Nunca & 1,0 \\
\hline Faixa etária & 8 & $30-60$ & $30-60$ & 1,0 \\
\hline Nível atividade & 10 & Leve & Leve & 1,0 \\
\hline Valor do VET & 10 & $2001-2400$ & $2001-2400$ & 1,0 \\
\hline \multicolumn{5}{|c|}{$\begin{array}{c}\text { VS global: }(3 * 0,2+3 * 1,0+8 * 1,0+10 * 1,0+ \\
10 * 1,0) / 3+3+8+10+10=0,92 \\
\end{array}$} \\
\hline
\end{tabular}

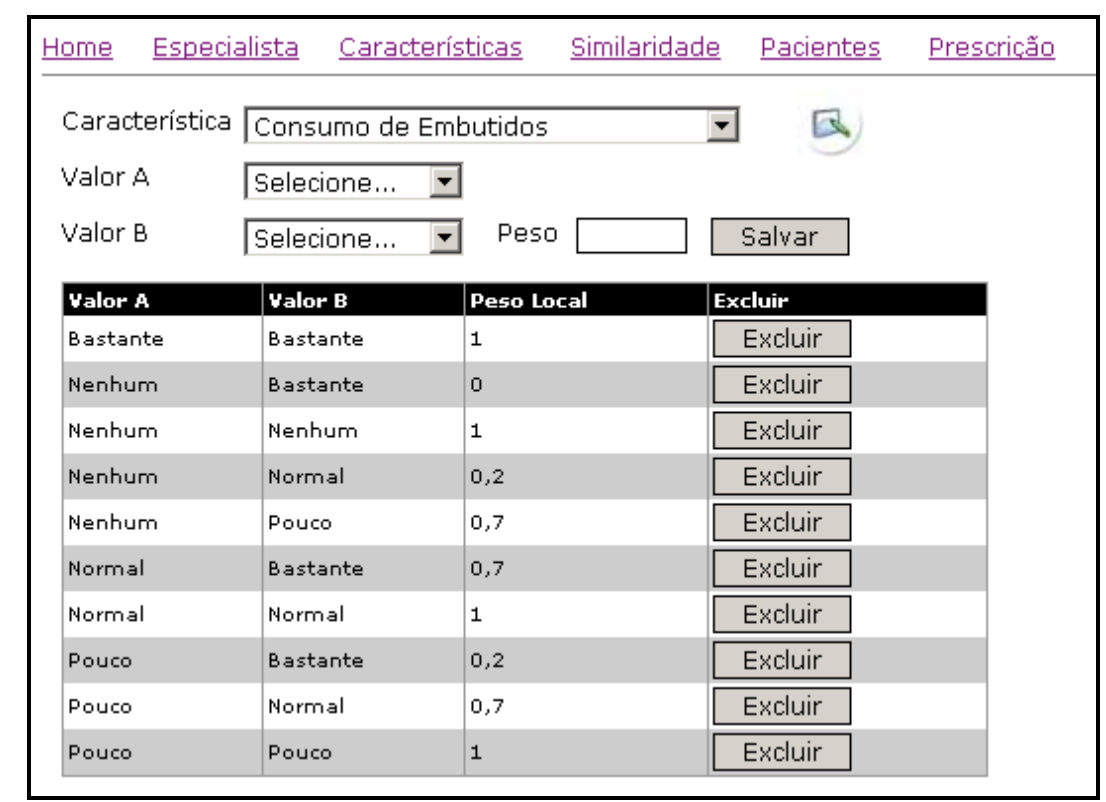

Figura 2 - Tela de Cadastro de Similaridade Local

\subsection{Reutilização, Revisão e Retenção}

No DietSysNet, utilizou-se da Adaptação Transformacional, que permite a adição e eliminação de atributos ou valores da solução.

$\mathrm{Na}$ recomendação de uma prescrição, o especialista pode alterar esta prescrição e inseri-la como um novo caso na base. Este processo representa o aprendizado do sistema.

\subsection{Implementação do Sistema}

Foram utilizadas as técnicas de engenharia de software como o diagrama de fluxo de dados, e entidade-relacionamento a fim de produzir uma modelagem consistente e de fácil adaptação a novos requisitos.

O código do sistema foi escrito em ASP .NET.- Active Server Pages que é uma tecnologia criada pela Microsoft para criar páginas de internet com conteúdo dinâmico. A escolha desta tecnologia permitiu explorar os recursos da internet, gerando uma interface simples e de fácil acesso. O caráter experimental permitiu utilizar uma base de dados Microsoft Access [16].

O DietSysNet foi estruturado em módulos separando-se as funcionalidades do nutricionista e do paciente. No módulo nutricionista, é possível criar os atributos da BC através do Cadastro de Características. As respostas pré-definidas de cada característica são 
mantidas através do Cadastro de Grupos de Valor. É possível fazer o cadastro de Pacientes, composto de informações básicas do paciente e das respostas a cada característica, formando assim seu perfil. A análise de Similaridade permite comparar os perfis dos pacientes, tornando-se muito útil para a recuperação e reutilização de tratamentos (prescrições) anteriores.

No módulo paciente, busca-se envolver o paciente no processo de acompanhamento para motivação, monitoramento e aceleração dos resultados obtidos com a dieta. O cadastro de acompanhamento permite um informe periódico do estado de saúde como: peso, sintomas observados, alergias, resultados de exames, além dos desvios alimentares. As consultas à prescrição e dados nutricionais dos alimentos consistem em valiosas fontes de informação para o paciente.

\subsection{Segurança das Informações}

Segundo o Conselho Federal de Nutricionistas é dever do nutricionista manter o sigilo no exercício da profissão sempre que tal seja do interesse dos indivíduos ou da coletividade assistida [3][4].

Com base neste princípio ético, e, considerando a forma de desenvolvimento do projeto, tornou-se necessário avaliar os requisitos relacionados à segurança da informação tanto no acesso do nutricionista que consulta os dados e históricos do paciente quanto ao paciente que realiza o cadastro de seus acompanhamentos.

Para tanto, há restrições de acesso, através de senhas e permissões, para garantir que pessoas não autorizadas tenham acesso aos dados.

\section{VALIDAÇÃo do Sistema}

O processo de validação visa avaliar se as soluções recomendadas pelo sistema são corretas ou não. Para isso, observa-se o comportamento do sistema diante da entrada simulada de casos. Estes casos são cuidadosamente preparados para analisar e testar a qualidade das soluções recomendadas pelo sistema.

A detecção das falhas deve conter as explicações do motivo de aquela solução não ser adequada para aquele problema. Tais explicações servirão para modificar a solução original do caso recuperado ou ainda, para corrigir os parâmetros que servem de base para o cálculo de similaridade entre os casos, razão em que geralmente se encontram deficiências.

A partir da necessidade de averiguar junto à nutricionista o resultado final do trabalho, foi realizada uma demonstração e assim como uma pequena bateria de testes. As conclusões e aprendizado obtidos estão apresentados a seguir.

Nos primeiros testes constatou-se que o sistema estava indicando como similares pacientes com hábitos e perfil muito diferentes do problema novo. De acordo com uma análise mais minuciosa, ficou evidente que isto acontecia em função dos pesos atribuídos às características que questionavam o histórico de patologias na família do paciente, inicialmente tomados como peso 6 (seis). O fato é que isto acabava por distorcer as avaliações, pois tais características apenas identificam a carga genética do paciente para a tomada de medidas preventivas e pouco deveriam interferir na comparação dos perfis de duas pessoas. Modificados estes parâmetros, ficou evidente a importância deste ajuste. As demais características também foram revistas e outros pesos sofreram ajustes, como consumo de saladas, que realmente identifica o comportamento do paciente e estava com um grau de importância muito baixo. Daí se conclui que é fundamental para um sistema RBC realizar ajustes com dados de teste, principalmente no peso dos atributos e nas medidas de similaridade locais entre valores de um mesmo atributo.

É importante registrar a dificuldade manifestada pelo nutricionista em executar esta configuração do sistema, o qual afirmou ficar confuso diante dos inúmeros conceitos apresentados e das possibilidades oferecidas. O que parece ter acontecido é uma dificuldade de enquadrar o modelo mental já utilizado ao algoritmo matemático, principalmente no que se refere aos pesos dos atributos e às similaridades locais.

Alguns testes evidenciaram algumas distorções do sistema e sua análise indicou a ausência da combinação de alguns valores de similaridade local. Este problema indicou a necessidade de desenvolvimento de algumas funcionalidades que facilitariam o uso do sistema. Para o cálculo de similaridade local, poderia haver uma rotina que combinasse todos os valores de resposta. Isto evitaria que alguma combinação fosse esquecida, reduzindo as chances de erro por insuficiência na parametrização. $\mathrm{Na}$ criação de uma nova característica também poderia haver um melhoramento se houvesse um mecanismo de preenchimento simplificado para os casos já existentes na base. Com a inclusão de novos pacientes, certamente aqueles que já tivessem este atributo preenchido deveriam ter seus valores corrigidos, evitando assim distorcer a recomendação do sistema.

Nos testes preliminares também houve problemas graves de desempenho do sistema (tempo de processamento e resposta), devido ao uso exagerado de recordsets. Em função disso, alguns códigos foram revistos, obtendo-se sensíveis ganhos. $\mathrm{O}$ fato de não se usar efetivamente a arquitetura cliente-servidor também comprometeu o desempenho do sistema, uma vez que tanto o servidor de internet quanto o cliente ocupavam a mesma estação. Como solução, a memória da máquina foi incrementada em $512 \mathrm{MB}$ de $R A M$, totalizando $768 \mathrm{MB}$.

Com quarenta casos cadastrados na BC, procedeu-se a validação final do sistema. Onze novos pacientes foram gerados para teste e nove casos foram reavaliados com a BC. No quadro 2 , estão descritos em detalhes os resultados obtidos pelo sistema.

Notou-se que em alguns novos casos, 
pacientes com baixa similaridade foram recomendados como solução. Isto se deve ao fato de que estes pacientes têm um perfil muito diferente daqueles atendidos no consultório. É o caso, por exemplo, de adultos magros ou com baixo peso que geralmente não buscam o auxílio de nutricionistas, ao contrário dos adolescentes, que mais preocupados com sua aparência acabam buscando estes serviços com mais freqüência.

Por outro lado, o número de pacientes adultos deste consultório que são hipertensos, ou diabéticos ou obesos ou ainda que possuam colesterol alto é mais freqüente e por isso a similaridade calculada acabou sendo superior. Desta observação, confirma-se a forte influência dos critérios utilizados para gerar a $\mathrm{BC}$ nos resultados obtidos com o um RBC.

Considerando-se os novos casos criados e os dados contidos no quadro 2, pode-se afirmar que:

1. Nas duas primeiras avaliações (casos 1 e 2 ), o sistema comparou pacientes que possuíam patologias. Segundo a análise do nutricionista, as diferenças quantitativas realmente não são grandes uma vez que seus VET's assemelhavam-se; entretanto, alguns tipos de alimentos, nunca poderiam fazer parte dos programas alimentares sugeridos. Notou-se que tais características deveriam ser tratadas como características de primeiro nível (vide Recuperação dos Casos). Feitas estas correções, novas avaliações foram feitas e os casos puderam ser então considerados parcialmente corretos, isto é, $18 \%$ dos casos, sofrendo pequenas adaptações;

2. Dos nove casos restantes, dois casos ou $18 \%$ foram considerados parcialmente corretos e sofreram pequenas alterações na quantidade dos alimentos, seja para mais ou para menos; os sete demais ou $64 \%$ dos casos, tiveram as prescrições recuperadas totalmente aproveitadas.

A avaliação do nutricionista indicou que os casos selecionados nos testes refletiram em grande parte as semelhanças reais entre os pacientes, concluindo-se que as condições para o reaproveitamento de uma prescrição foram satisfeitas, atestando-se portanto, a assertividade do sistema.

Considerando-se agora a reavaliação feita sob os casos já existentes e os dados contidos no quadro 2 , pode-se afirmar que:

1. Cinco casos $(13,14,15,18,20)$ apresentaram um grau de similaridade elevado, porém necessitariam ainda de adaptações para aproveitamento total. Este número representa $56 \%$ dos casos e foi considerado preocupante pelo nutricionista, considerando seus índices de similaridade. A dificuldade em ajustar os parâmetros reproduzindo os mesmos efeitos deixou claro que independente do sistema, é sempre fundamental que o especialista faça a análise das sugestões dadas pelo sistema.

2. Quatro casos foram considerados totalmente reaproveitáveis, representando $44 \%$ dos casos. 
Quadro 2 - Resultados dos testes efetuados

\begin{tabular}{|c|c|c|c|c|c|}
\hline No & Caso Novo & Reutilizado & Caso & Comentário & $\begin{array}{c}\text { VS } \\
\text { global }\end{array}$ \\
\hline 1 & Luciana F. & Não & - & $\begin{array}{l}\text { A alimentação para diabéticos é diferenciada; } \\
\text { Após alterar primeiro nível, pequenos ajustes } \\
\text { foram feitos. }\end{array}$ & 79,26 \\
\hline 2 & Sérgio F. & Não & - & $\begin{array}{l}\text { A ausência de recomendações essenciais para } \\
\text { hipertensos considerou-se incorreta; Após } \\
\text { modificação, também foram aceitas com pequenos } \\
\text { ajustes. }\end{array}$ & 82,88 \\
\hline 3 & Fabíola B. & Sim & Vânia M. & & 88,04 \\
\hline 4 & Maria C. & Sim & Zeli M. & & 80,37 \\
\hline 5 & Carlos A. & Sim & João V. & & 90,37 \\
\hline 6 & Daiani L. & Sim & $\begin{array}{l}\text { Camila } \\
\text { P. }\end{array}$ & & 87,48 \\
\hline 7 & Luciano F. & Não & & $\begin{array}{l}\text { Os VETs são diferentes; foi necessário acréscimo } \\
\text { de Kcal }\end{array}$ & 89,02 \\
\hline 8 & Rosângela & $\mathrm{Sim}$ & $\begin{array}{l}\text { Claudia } \\
\text { P. }\end{array}$ & & 79,03 \\
\hline 9 & Danilo R. & Sim & Luis V. & & 88,42 \\
\hline 10 & Felipe W. & Sim & Maciel P. & & 84,67 \\
\hline 11 & Fátima P. & Não & Iraci & $\begin{array}{l}\text { Os VETs são diferentes; foi necessário de } \\
\text { acréscimo de Kcal }\end{array}$ & 71,88 \\
\hline 12 & BC Daciane & Sim & Gabriela & & 85,64 \\
\hline 13 & BC Luis A. & Não & Luciano & Ajuste de quantidades de alimentos & 89,02 \\
\hline 14 & BC João P. & Não & - & $\begin{array}{l}\text { Ajuste de quantidades de alimentos e } \\
\text { recomendações }\end{array}$ & 82,45 \\
\hline 15 & BC Julia & Não & - & $\begin{array}{l}\text { Os VETs são diferentes; necessita de acréscimo de } \\
\text { Kcal }\end{array}$ & 82,21 \\
\hline 16 & BC Madalena & Sim & Zeli M. & $\begin{array}{l}\text { Os VETs são diferentes; necessita de decréscimo } \\
\text { de Kcal }\end{array}$ & 80,82 \\
\hline 17 & BC Sérgio $\mathrm{C}$. & Sim & Paulo F. & & 78,96 \\
\hline 18 & BC Paulo & Não & João P & & 82,45 \\
\hline 19 & BC Carla C. & Sim & Roseli & & 85,21 \\
\hline 20 & BC Matilde & Não & Zeli M. & $\begin{array}{l}\text { Apesar dos VETs serem iguais, a paciente Matilde } \\
\text { é diabética; alguns alimentos devem ser } \\
\text { substituídos }\end{array}$ & 74,54 \\
\hline
\end{tabular}




\section{Trabalhos Corelatos}

Com o objetivo de obter informações sobre outros trabalhos da área, procurou-se por sistemas existentes através de websites ou revistas da área. Encontraram-se os que seguem sendo os dois primeiros baseados em [1].

1. CHEF [7] desenvolve novos pratos a partir de outros. São solicitados os objetivos e então se procura satisfazer estes, recuperando do banco a receita que atenda a maior parte dos objetivos e cria-se uma nova receita para a situação apresentada.

2. JULIA [8] trabalha com planejamento de refeições. Utiliza-se de casos que a partir das restrições propostas guiam à elaboração de uma nova refeição.

3. DIETWIN [5] sistema de avaliação nutricional que oferece vários produtos: clínico, profissional, rótulo de alimentos e análise nutricional mas não foram encontrados comentários sobre a reutilização de experiências.

\section{CONClusão}

Com a realização deste trabalho foi possível avaliar os benefícios que a aplicação de um sistema RBC proporciona, além de uma valiosa experiência prática desta técnica.

Pode-se avaliar ainda, o alto grau de abrangência na aplicação destes sistemas. Diversas áreas e domínios podem ser contemplados com suas ferramentas, trazendo muitas vantagens e gerando soluções com potencial qualidade.

A validação do sistema com casos reais de teste geraram resultados satisfatórios na opinião do especialista, levando à conclusão de que o sistema estaria apto para ser utilizado em consultórios.

De modo geral, conclui-se que a implementação de um sistema RBC para o consultório foi totalmente válida e produziu resultados satisfatórios, mas não excluiu a interação humana.

\section{Referências Bibliográficas}

[1] ABEL, Mara. Um estudo sobre o Raciocínio Baseado em Casos. Porto Alegre: Universidade Federal do Rio Grande do Sul, 1996.

[2] BARRETO, J.M. Inteligência Artificial - No limiar do século XXI Abordagem híbrida, simbólica, conexionista e evolutiva. $3^{\mathrm{a}}$. Edição, $\rho \rho \rho$ Edições, Florianópolis, 2001.

[3] CFN - Conselho Federal de Nutricionistas. Código de Ética do Nutricionista.
A agilidade e a confiabilidade podem ser facilmente obtidas em uma aplicação RBC criando-se adequadamente a $\mathrm{BC}$. Isso contribui para o satisfatório acúmulo e estruturação de conhecimento, bem como permite extrair maiores ganhos sob as experiências retidas.

A escolha da tecnologia ASP.NET como linguagem trouxe ganhos ao projeto como o acesso a aplicação de qualquer ponto exigindo apenas uma conexão de Internet e aproveitando a segurança existente dos provedores de hospedagem de sítios.

$\mathrm{O}$ nutricionista, que neste caso é o usuário da aplicação RBC, pode contar com um sistema que lhe dá maior tranqüilidade na determinação de solução. Isto porque $\mathrm{o}$ sistema utiliza ponderações e regras embasadas no próprio conhecimento do especialista e na realidade em que foram modeladas no sistema, pois um grande número de fatores são considerados durante a avaliação de similaridade.

O grau de automatização resultante na geração da solução reduziu o esforço do nutricionista. Esta economia de esforços permite a ele dedicar-se ao estudo e aperfeiçoamento dos diagnósticos. Este ciclo promove o aperfeiçoamento do conhecimento acumulado.

Analisando o DietSysNet, percebe-se pela modelagem dinâmica do problema que o sistema poderia ser aplicado a outros domínios. A possibilidade de utilizá-lo para outras aplicações, em princípio, decorre na adaptação do modelo de solução, contemplando as áreas em que for utilizado.

O desenvolvimento de uma aplicação baseada em web pode mudar sensivelmente as formas de comercialização dos serviços de Nutrição. Novas funcionalidades poderão ser oferecidas através do sistema, transformando o DietSysNet num sítio de serviços de nutrição.

A funcionalidade de Compensação de Dietas foi apontada como de grande potencial pelo nutricionista, constituindo-se em um forte candidato para trabalhos futuros. Vale lembrar que nas pesquisas realizadas no mercado, nenhum sistema encontrado dispõe deste recurso, caracterizando-se como um diferencial.

Brasília, 10/5/2004.

[4] CFN - Conselho Federal de Nutricionistas. Revista. Edição de janeiro/fevereiro/março/abril de 2002 Ano III $N^{\circ} 6,15 p$.

[5] DIETWIN. Software de avaliação nutricional. Disponível em: <http:// http://www.dietwin.com.br>. Acesso em: 26 de julho de 2006.

[6] EVALD, Gregory A. Manual de Terapêutica Clínica. 28 ${ }^{\mathrm{a}}$ Ed. Guanabara 
Koogan, 1996.

[7] HAMMOND, K. J. CHEF: a model of case based planning. AAAI-86. Procedings. AAAI Press/MIT Press. Cambridge, MA, 1986 apud KOLODNER 1993.

[8] HINRICHS, T R. Problem Solving in Open Worlds: A Case Study in Design. Lawrence Erlbaum Associates, 1992.

[9] JONES, A. Russell. ASP 3 Active Server Pages 3 "A Bíblia". Ed. Makron Books, 2001, 748p.

[10] KOLODNER, J. L. Case-Based Reasoning. Morgan Kaufmann Pub., Inc. 1993.

[11] LINDEMANN, Vanessa. Raciocínio baseado em casos In: BARONE, Dante Augusto Couto (Org.). Sociedades artificiais: a nova fronteira da inteligência nas máquinas. Porto Alegre: Ed Bookman, 2003. p. 209-229.

[12] LORENZI, F \& ABEL, M. Aplicando raciocínio baseado em casos $n$ a investigação de irregularidades nas internações hospitalares. Porto Alegre: PGCC, 1998.

[13] LORENZI, F \& ABEL, M. Aplicando raciocínio baseado em casos na investigação de irregularidades nas internações hospitalares.In Congresso Brasileiro de Computação, Itajaí, 2002.

[14] LORENZI, Fabiana. Disciplina de
Inteligência Artificial. Canoas: Universidade Luterana do Brasil, 2005.

[15] MASIERO, Orzeli M., Trabalho de Conclusão em Nutrição - Hábitos Alimentares, Avaliação Nutricional e doenças não transmissíveis. São Leopoldo: Universidade de Vale do Rio dos Sinos, 2001.

[16] PRATES, Rubens. ASP - Guia de consulta rápida. São Paulo: Ed. Novatec, 2000, 128p.

[17] SILVA, Mara Reis, NAVES, Maria Margareth Veloso. Manual de nutrição e dietética. Goiânia: Ed. Cegraf - UFG, 1994. 151p.

[18] TUCUNDUVA, Sonia. Nutrição e Técnica Dietética . São Paulo: Ed. Manole, 2003. 390p.

[19] UOL. Boa Saúde, Belo Horizonte. Disponível em: $<$ http://boasaude.uol.com.br $>$. Acesso em: 18 e 19 de abril de 2005.

[20] WANGENHEIM, Christiane Gresse Von; WANGENHEIM, Aldo von. Raciocínio baseado em casos. Barueri, SP: Ed Manole, 2003. 293p.
Intolisanmois A ntifiniol Connon. 